\title{
- Incidence of Deep Venous Thrombosis Followed by Fracture of Neck of Femur in the Elderly Patients with Prophylaxis of Anticoagulant: A Cross-Sectional Study

\section{Syed Muhammad Khalid Karim ${ }^{1}$, Niaz Hussain Keerio' Sajjad Hussain Bhatti ${ }^{3}$, Zahoor Illahi Soomro ${ }^{4}$, Jamil Ahmed Khoso5, Ghazanfar Ali Shah ${ }^{6}$}

\begin{abstract}
'Assistant Professor Orthopedic, Dow Medical College \& Dr. Ruth K.M. Pfau Civil Hospital Karachi, Pakistan; ${ }^{A}$ Assistant Professor Orthopedic, Muhammad Medical College and Hospital Mirpurkhas, Pakistan; ${ }^{3}$ Assistant Professor Orthopedics, Pir Abdul Oadir Shah Jillani Institute of Medical Sciences GIMS Gamabat, Pakistan; ${ }^{4}$ Associate Professor Orthopedic,Peoples University of Medical \& Health Sciences Nawabshah, Pakistan; ${ }^{5}$ Senior Registrar Orthopedic, Shaheed Mohtarma Benazir Bhutto Medical Collage and Lyari General Hospital Karachi, Pakistan; ${ }^{6}$ Assistant Professor Orthopedics, SMBBIT/Dow University of Medical and Health Sciences Karachi, Pakistan.
\end{abstract}

\section{ABSTRACT}

Introduction: Fracture of the neck of the femur is the most common fracture due to osteoporosis in the elderly population. Almost one-third of the patients with such fractures lead to mortality.

Aim: To determine the incidence of deep vein thrombosis in elderly patients with a fracture in the head of femur and prophylactic administration of anticoagulants.

Methodology: Elderly patients (age above 60 years) with fractures in the head of the femur were included in this study. They should have either extracapsular or extracapsular fracture of the neck of the femur. According to the exclusion criteria, patients who were below 60 years old and have a fracture of the neck of the femur but also had multiple traumas and a previous history of deep vein thrombosis. All patients who were non-compliant to the prophylactic drugs were also categorized in exclusion criteria. The patients were administered prophylactic anticoagulants on admission. All the patients were investigated through Color Doppler Ultrasonography when they were admitted for the confirmation and documenting the diagnosis.

Result: Total 60 patients standing the inclusion criteria were selected. All of them were above the age of 60 years. They all were given thromboprophylaxis. Total 12 out of 60 patients presented with deep vein thrombosis.

Conclusion: Deep vein thrombosis is quite common in patients with fractures of the head of the femur and they must be given anticoagulation prophylactically. In the present study, all patients received the drug enoxaparin for the prevention of DVT, however, significant number of patients still present with the deep vein thrombosis.

Key Words: Deep vein thrombosis, Anticoagulant, Fracture of head of femur, Thromboprophylaxis, Color Doppler Ultrasonography, diagnosis

\section{INTRODUCTION}

Fracture of the neck of the femur is the most common fracture due to osteoporosis in the elderly population. Almost one-third of the patients with such fractures lead to mortality. Due to comorbidities such as hypertension, peripheral vascular diseases, heart diseases, diabetes, etc., these patients are prone to face thromboembolic events and participate in poor prognosis. ${ }^{1}$ Deep vein thrombosis prolongs the hospital stay of the patient, overburdens the patient regarding the cost of the treatment and leads to venous insufficiency. Sometimes deep vein thrombosis also causes postphlebitic syndrome. Pulmonary embolism is the lethal most complication of deep vein thrombosis which is the result of dislodging of a clot in the vessels and impairment of circulation of blood towards pulmonary arteries. The more common source of pulmonary embolism is deep vein thrombosis of the proximal lower extremity.

\section{Corresponding Author:}

Niaz Hussain Keerio, Assistant Professor Orthopedic, Muhammad Medical College and Hospital Mirpurkhas, Pakistan. Email: niaz_h@hotmail.com

ISSN: $2231-2196$ (Print)

ISSN: $0975-5241$ (Online)

Received: 13.03 .2021

Revised: 22.04 .2021

Accepted: 03.05.2021

Published: 04.06 .2021 
In orthopedic patients, common contributing fractures in deep vein thrombosis are that of acetabulum fractures and pelvic fractures. Total knee replacement surgery and total hip replacement surgery can also lead to deep vein thrombosis postoperatively. ${ }^{2}$ However, the incidence of deep vein thrombosis is higher in poly-trauma patients and hip fracture patients.

The diagnosis of deep vein thrombosis can be made easily by assessing and examining the patient physically. In deep vein thrombosis, swelling is seen in only one leg. The affected area feels warm. The color of the affected leg is either reddish or bluish. The clinical signs of deep vein thrombosis are a clear indication of its diagnosis, however, this criteria is not always reliable and imaging techniques are required for confirmation. Color Doppler magnetic resonance venography is the most specific imaging test to confirm the diagnosis of deep vein thrombosis. It is so far the gold standard method. Other laboratory investigations such as D-dimers and complete blood pictures are also needed. Treatment of deep vein thrombosis includes the use of compression stockings, blood thinners, and venous filters. Blood thinners can also be used as prophylactic agents. ${ }^{3}$ Prophylaxis through anticoagulants has been greatly helpful in patients with fractures of the head of the femur.

The attention of the orthopedists has been diverted to the prophylaxis of DVT and treatment of DVT by different means. One of the most effective methods to get rid of DVT is considered to be prevention through drugs. Low molecular weight heparin has been effective in this regard. The incidence of DVT in trauma patients ranges from $11 \%$ to $33 \%$ in some studies. ${ }^{4}$ On the other hand, DVT can also be found in the uninjured limb. Hypercoagulation state remains thereafter at least one month of the injury in $80 \%$ of the patients. ${ }^{5}$ However, the incidence is more in the injured limb. It is also noted that $75 \%$ of all the patients with DVT are asymptomatic. $^{6}$

The objective of this study is to evaluate the incidence of occurrence of deep vein thrombosis in patients who have been given anticoagulants after they had a fracture in the neck of the femur. The patients considered are elderly.

Study design: Cross-sectional study

Place and duration: This study was conducted at Dow Medical College \& Dr. Ruth K.M. Pfau Civil Hospital Karachi, Pakistan from June 2020 to June 2021.

Methodology: A total of 60 patients were considered in this research. Permission was taken from the ethical review committee of the institute. All of the patients were selected based on inclusion criteria designed for the research. According to the inclusion criteria, the subjects included should be above 60 years of age. They should have an either intracapsular or extracapsular fracture of the neck of the femur. The comorbidities should be reported if any. An exclusion criterion was also designed to omit maximum errors from the research. According to the exclusion criteria, patients who were below 60 years old and have a fracture of the neck of the femur but also had multiple traumas and a previous history of deep vein thrombosis. All patients who were non-compliant to the prophylactic drugs were also categorized in exclusion criteria.

The protocol of the study was set in such a way that the patients were administered prophylactic anticoagulants on admission. The dose was started with an injection of enoxaparin $40 \mathrm{mg}$. The drug was given subcutaneously once daily. The drug was prescribed for 2 weeks. The recommendations of the American College of Clinical Pharmacy were followed for the prophylaxis. According to the recommendations of ACCA, the drug should be stopped 12 hours before surgery and likewise, it should be continued 12 hours after the surgery.

All the patients considered in the study were investigated through Color Doppler Ultrasonography when they were admitted for the confirmation and documenting the diagnosis. A thorough physical examination was done at the time of admission. Other pre-operative protocols such as assessment of major surgical profile, physician fitness, cardiologic fitness, and anesthesia fitness were posted for surgery. In our hospital, the major surgical profile includes baseline investigations viz. complete blood picture, blood sugar, blood grouping, $\mathrm{Rh}$ factor, renal function test, liver function test, serum electrolytes, HBsAg, AntiHCV, Urine complete examination, Chest $\mathrm{X}$-ray, and ECG. Moreover, 2D-echo is also done if the cardiologist advises. Serial Color Doppler Ultrasonography is done and the results are recorded on standard Performa. All this data is saved on the proper software that is SPSS version 22. The imaging was also done 4 days after the surgery. A follow-up check-up was done 13 days after the surgery in which the USG was repeated. During the study, Color Doppler USG was attempted on the admitted patient on the $12^{\text {th }}$ and $13^{\text {th }}$ postoperative days as well. Results were collected, analyzed, and compiled in tabulated and graphical manners.

\section{RESULTS}

The sixty patients with the head of the femur fracture considered in the research were subdivided into three groups based on age. Following is the division of the groups: Group A consisted of patients with ages ranging from 60 to 70 years. This group had 40 patients (66.66\%). Group B consisted of patients with ages ranging from 70 to 80 years. This group had 16 patients $(26.66 \%)$. Group C consisted of patients with age above 80 . This group only had 4 cases $(6.66 \%$ ) (As shown in table 1) Out of all the fractures reported in this age group in the research, 35 cases were of fracture of neck of 
femur $(58.33 \%)$ and that of intertrochanteric fracture were 25 (41.67\%) (As shown in table 2).

Out of all the causes of these fractures, deep vein thrombosis was seen more in the patients who had a fracture in the neck of the femur. Out of 35 cases of fracture of neck of femur, 9 patients presented with deep vein thrombosis $(25.71 \%)$. Total 3 patients from the 25 patients who had an intertrochanteric fracture, suffered from deep vein thrombosis (12\%) (As shown in table 3 ). Total 12 patients from the 60 patients who had suffered from the fracture of the head of the femur, presented with DVT even after thromboprophylaxis. (As shown in table 4)

\begin{tabular}{|c|c|c|c|}
\hline Group & Age (Years) & Number & Percentage \\
\hline Group A & $60-70$ & 40 & 66.66 \\
\hline Group B & $70-80$ & 16 & 26.66 \\
\hline Group C & Above 80 & 4 & 6.66 \\
\hline Total & & & 60 \\
\hline
\end{tabular}

Table 2: Type of fractures with the percentage

\begin{tabular}{lcc} 
Type of Fracture & Number & Percentage \\
Fracture of neck of femur & 35 & 58.33 \\
Intertrochentric fracture & 25 & 41.67 \\
\hline
\end{tabular}

Table 3: Deep Venous Thrombosis in patients with a fracture in the head of the femur

\begin{tabular}{lccc} 
Type of Fracture & No. of cases & $\begin{array}{c}\text { Patients } \\
\text { with } \\
\text { DVT }\end{array}$ & $\begin{array}{c}\text { No. of } \\
\text { patients with } \\
\text { DVT }\end{array}$ \\
$\begin{array}{l}\text { Fracture of neck } \\
\text { of femur }\end{array}$ & 35 & 9 & $25 \cdot 71 \%$ \\
$\begin{array}{l}\text { Intertrochentric } \\
\text { fracture }\end{array}$ & 25 & 3 & $12 \%$ \\
\hline
\end{tabular}

Table 4: DVT in patients who were given anticoagulation prophylactically

\begin{tabular}{lcc}
$\begin{array}{l}\text { Number of } \\
\text { cases }\end{array}$ & $\begin{array}{c}\text { DVT positive after throm- } \\
\text { boprophylaxis }\end{array}$ & Percentage \\
60 & 12 & $20 \%$ \\
\hline
\end{tabular}

\section{DISCUSSION}

In the present study, the number of patients between the age of 60 years and 70 years is $40(66.66 \%)$, between the age of 70 years and 80 years is $16(26.66 \%)$, and above the age of 80 years are $4(6.66 \%)$. A similar study was conducted by Bengao et al., in 2018 in which the average age of the patients was 75 years. ${ }^{7}$ Another such study was carried out by Xing et al.,the range of patients in this study was also above
60 years. ${ }^{8}$ According to the study of Niu et al., most of the patients who present with DVT in the trauma unit were elderly. ${ }^{9}$ A similar study carried out by Zhao et al., also proves that most of the patients suffering from deep vein thrombosis followed by a fracture in the head of the femur are above the age of 65 years. ${ }^{10}$ The study of Zhang et al., conducted a study on deep vein thrombosis in both extremities after fracturing of the hip joint. His study included 463 patients and all of them were elderly. ${ }^{11} \mathrm{Fu}$ et al., conducted a study in which he kept a special focus on the administration of anticoagulants. The inclusion criteria regarding age were kept in such a way that the age of the patients should be greater than 16 years. However, the average age of patients with deep vein thrombosis after hip fracture was 71.3 years. ${ }^{12}$ Hence, it is evident from multiple studies that deep vein thrombosis is a common complication of trauma in the hip joint resulting in fracture of the head of the femur.

In this study, a total of 60 persons were considered to have a fracture of the head of the femur. Total 35 patients presented with a fracture in the neck of the femur which is $58.33 \%$. The remaining 25 had intertrochanteric fracture which is $41.67 \%$ of the total patients. In the study of Bengoa et al., his main motive to conduct the study was to see the prevalence of deep vein thrombosis in fracture patients. Prophylaxis of anticoagulation was not given. The study concludes that $17.1 \%$ of patients present with deep vein thrombosis if medical care is not given within 48 hours after the fracture. The study also suggests screening every fracture patient for deep vein thrombosis. ${ }^{7}$ The study of Xing et al., is based on elderly patients with deep vein thrombosis after fracturing in China. According to the study, the predictors of the risk factor in such patients are the age of the patient, gender of the patient, time from injury to hospitalization, levels of D-dimers, and fibrinogen. ${ }^{8}$ These factors are helpful in the prediction of dosage of anticoagulants and prognosis of the treatment.

Niu et al., investigated the epidemiological character of deep vein thrombosis in elderly patients. He compared the rate of DVT prevalence in preoperative patients and found that it is not of much significance. However, he drew attention to the uninjured extremity and described that there are very evident chances of DVT in the uninjured leg. ${ }^{9}$ The study carried out by Zhao et al., is similar to the present study in terms of prevention through anticoagulants. In his study, pre-operative Doppler ultrasound was advised for both limbs. They found that $10.2 \%$ of patients had developed deep vein thrombosis preoperatively..$^{10}$ The study of Zhang et al., suggests that all the researches that have been done in this regard, underestimate the incidence of deep vein thrombosis in hip fracture elderly patients. They also focused on the occurrence of DVT in both extremities. ${ }^{11}$ According to the study of Fu et al., orthopedists should not take the occurrence of DVT sparingly as this is a common complication of a hip fracture. ${ }^{12}$ 
In the present study, 60 patients received the drug enoxaparin for the prevention of DVT, however, 12 patients $(20 \%)$ still present with the disease. Color Doppler ultrasound was used for the detection of DVT.

\section{CONCLUSION}

In patients with a fracture in the head of the femur, the incidence of deep vein thrombosis is quite high. Prevention of deep vein thrombosis can be done by the administration of an anticoagulant. In the present study, all patients received the drug enoxaparin for the prevention of DVT, however, significant number of patients still present with the deep vein thrombosis. The most reliable and convenient method of screening DVT is Color Doppler Ultrasonography. Comorbidities and the age of the patient make them special in terms of physiological complications. Hence, they need special treatment as well. Due to the high rate of DVT in elderly patients with a fracture in the head of the femur, thromboprophylaxis has become essential.

\section{ACKNOWLEDGEMENT}

Authors acknowledge the immense help received from the scholars whose articles are cited and included in references of this manuscript. The authors are also grateful to authors / editors / publishers of all those articles, journals and books from where the literature for this article has been reviewed and discussed

\section{Permission:}

It was taken from the ethical review committee of the institute:

\section{Source of funding:}

None

\section{Conflict of interest:}

None
Authors' Contribution: All authors' mentioned in the manuscript contributed to the study.

\section{REFERENCES}

1. Cauley JA, Chalhoub D, Kassem AM, Fuleihan GE. Geographic and ethnic disparities in osteoporotic fractures. Nature Reviews Endocrinology. 2014; 10(6):338-51.

2. Wolberg AS, Rosendaal FR, Weitz JI, Jaffer IH, Agnelli G, Baglin $\mathrm{T}$, et al. Venous thrombosis. Nature reviews Disease primers. $2015 ; 1(1): 1-7$.

3. Zamora T, Klaber I, Urrutia J. Hip fracture in the elderly. Clinical Medicine Insights. Geriatrics. 2017; 10:1-0.

4. Mears SC, Kates SL. A guide to improving the care of patients with fragility fractures, edition 2 . Geriatric orthopaedic surgery \& rehabilitation. 2015; 6(2):58-120.

5. Jaff MR, McMurtry MS, Archer SL, Cushman M, Goldenberg N, Goldhaber SZ, Jenkins JS, Kline JA, Michaels AD, Thistlethwaite $\mathrm{P}$, Vedantham S. Management of massive and submassive pulmonary embolism, iliofemoral deep vein thrombosis, and chronic thromboembolic pulmonary hypertension: a scientific statement from the American Heart Association. Circulation. 2011; 123(16):1788-830.

6. Kearon C, Akl EA. Duration of anticoagulant therapy for deep vein thrombosis and pulmonary embolism. Am. J. Hematol. 2014; 123(12):1794-801.

7. Bengoa F, Vicencio G, Schweitzer D, Lira M, Zamora T, Klaber I. High prevalence of deep vein thrombosis in elderly hip fracture patients with delayed hospital admission. European Eur. J. Trauma Emerg. Surg. 2018; 46(4):913-917.

8. Xing F, Li L, Long Y, Xiang Z. Admission prevalence of deep vein thrombosis in elderly Chinese patients with hip fracture and a new predictor based on risk factors for thrombosis screening. BMC Musculoskeletal Disorders. 2018; 19(1).

9. Niu S, Li J, Zhao Y, Ding D, Jiang G, Song Z. Preoperative deep venous thrombosis (DVT) after femoral neck fracture in the elderly, the incidence, timing, location and related risk factors. BMC Musculoskeletal Disorders. 2021; 22(1).

10. Zhao K, Wang Z, Tian S, Hou Z, Chen W, Zhang Y. Incidence of and risk factors for pre-operative deep venous thrombosis in geriatric intertrochanteric fracture patients. International Orthopaedics. 2021.

11. Noor SS, Hussain N, Javed I. Outcome of Hemiarthroplasty in elderly patients with fracture neck of femur. Journal of Pakistan orthopedic association (JPOA). feb 2010; 22 (1):14- 19.

12. Noor SS, Keerio NH, Valecha NK, Qureshi MA. J Biochem Tech (2019) 11(3): 92-96. 